\title{
Sensory Versus Cognitive Components in Harmonic Priming
}

\author{
Emmanuel Bigand and Bénédicte Poulin \\ Université de Bourgogne
}

\author{
François Madurell \\ Université Paris-Sorbonne
}

\author{
Barbara Tillmann \\ Dartmouth College
}

Daniel A. D’Adamo
Université de Bourgogne

\begin{abstract}
This study investigated the strength of sensory and cognitive components involved in musical priming. In Experiment 1, the harmonic function of the target chord and the number of pitch classes shared by the prime sequence and the target chord were manipulated. In Experiment 2, the temporal course of sensory and cognitive priming was investigated. For both musician and nonmusician listeners, cognitive priming systematically overruled sensory priming even at fast and very fast tempi (300 ms and $150 \mathrm{~ms}$ per chord). Cognitive priming continued to challenge sensory priming processes at extremely fast tempo $(75 \mathrm{~ms}$ per chord) but only for participants who began the experimental session with slower tempi. This outcome suggests that the cognitive component is a fast-acting component that competes with sensory priming.
\end{abstract}

A musical context generates expectancies about upcoming musical events in listeners. Several features govern expectancy formation in Western music, including melodic interval size and melodic contour (Boltz \& Jones, 1986; Cuddy \& Lunney, 1995; Krumhansl, 1995; Schellenberg, 1996), rhythm (Boltz, 1993; Jones, Boltz, \& Klein, 1993), and the harmonic structure of the musical context (Bharucha \& Stoeckig, 1986, 1987). The present study focuses on priming resulting from the last factor, referred to as harmonic priming.

As illustrated in Figure 1, both sensory processes and cognitive processes potentially govern harmonic priming. A context may prime the processing of chords that share harmonic spectra (i.e., same component tones, or overtones) with it (referred to as sensory priming). By contrast, a context may prime the processing of chords that are related to it according to the rules of a given musical idiom. This form of priming is based on the activation of knowledge about the musical idiom and is referred to as cognitive priming. These two accounts of harmonic priming are not incompatible, and it can be assumed that both are combined in natural listening situations. The respective importance of the two types of priming is likely to depend on other factors, such as musical expertise or the speed at which the musical context is presented. The purpose of the present study was to address this issue in two experiments. In Experiment 1, we contrasted cognitive priming

Emmanuel Bigand, Bénédicte Poulin, and Daniel A. D’Adamo, Laboratoire d'Étude de l'Apprentissage et du Développement, Centre National de la Recherche Scientifique (LEAD-CNRS), Université de Bourgogne, Dijon, France; Barbara Tillmann, Department of Psychological and Brain Sciences, Dartmouth College; François Madurell, Department of Musicology, Université Paris-Sorbonne, Paris, France.

This research was supported in part by a grant from the International Foundation for Music Research and by the CNRS's Cognition and Information Processing program.

Correspondence concerning this article should be addressed to Emmanuel Bigand, Université de Bourgogne, LEAD-CNRS, 6 Boulevard Gabriel, 21000 Dijon, France. E-mail: emmanuel.bigand@u-bourgogne.fr with sensory priming by simultaneously manipulating the harmonic function of the target chord and the number of notes and pitch classes shared by the prime sequence and the target chord. In Experiment 2, we investigated the time course of cognitive and sensory priming by varying the tempo of the prime context. Both experiments were run with musically naive and expert listeners. Before presenting the experiments, we consider the two theoretical accounts in more detail.

\section{A Cognitive Account of Harmonic Priming}

In the Western tonal system, 12 pitch classes (referred to with the labels C, C\# or Db, D, D\# or Eb, E, F, F\# or Gb, G, G\# or Ab, $\mathrm{A}, \mathrm{A} \#$ or $\mathrm{B} b$, and $\mathrm{B}$ ) are organized in subsets of seven tones resulting in 12 major and 12 minor keys. In a given key, seven chords (major, minor, or diminished) can be defined, each on a different degree of the scale. Between these chords there exists a harmonic hierarchy: Chords built on the first, fourth, and fifth degrees of the scale (referred to as tonic, subdominant, and dominant chords, noted as I, IV, and V, respectively) are the most important chords of the key, the tonic chord being the most referential one. For example, in the $\mathrm{C}$ major key, the $\mathrm{C}$ chord (consisting of the tones $\mathrm{C}, \mathrm{E}$, and $\mathrm{G}$ ), $\mathrm{F}$ chord (consisting of the tones $\mathrm{F}, \mathrm{A}$, and $\mathrm{C}$ ), and $\mathrm{G}$ chord (consisting of the tones $\mathrm{G}, \mathrm{B}$, and D) act as tonic, subdominant, and dominant chords, respectively. In the present study, we focus on the small difference in musical functions between the tonic and subdominant chords. Both chords belong to the same context key and act as strong cognitive reference points in Western tonal music (Bharucha \& Krumhansl, 1983; Lerdahl, 1988).

The Western harmonic hierarchy is thought to be internalized by Western listeners through mere exposure to Western music (Francès, 1958/1988; Krumhansl, 1990a; Tillmann, Bharucha, \& Bigand, 2000). A cognitive approach to harmonic priming stipulates that a context activates the listener's knowledge of the Western harmonic hierarchy, resulting in faster processing of a target chord that is more referential in the present key context. Bharucha 


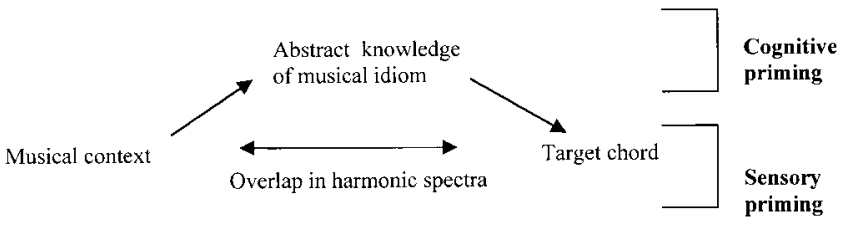

Figure 1. Cognitive (top) and sensory (bottom) accounts of harmonic priming.

and Stoeckig $(1986,1987)$ observed that the processing of a target chord depends on its harmonic relationship with the prime chord. In-tune target chords are processed faster when they share a parent key with the prime chord (as $\mathrm{C}$ and $\mathrm{G}$ major chords) rather than when they do not (as $\mathrm{C}$ and $\mathrm{F} \#$ major chords). Comparable priming effects have been reported in long musical contexts (Bigand, Madurell, Tillmann, \& Pineau, 1999; Bigand \& Pineau, 1997; Tillmann \& Bigand, 2001; Tillmann, Bigand, \& Pineau, 1998). In Bigand and Pineau (1997), eight-chord sequences were presented to the participants. The target chord-the last chord in the sequence-acted as either a stable tonic chord or a musically congruent but less stable subdominant chord. The tonic target was assumed to be more expected than the subdominant target. The results showed that priming in long contexts is similar to priming in short contexts: Tonic chords were processed faster and more accurately than subdominant chords. These results support the hypothesis of an anticipatory processing due to musical expectancies at a cognitive level. The processing of the most harmonically related chord is facilitated because the processing is primed by the previous context before the chord actually sounds. Cognitive priming effects in music share similarities with word and sentence priming effects observed in language (Tillmann \& Bigand, 2001, in press). In both cases, the processing of a target event is mediated by the activation of an abstract mental representation: The more the target is related to the previous context in light of this abstract representation, the more its processing is facilitated.

\section{Sensory Accounts of Harmonic Priming}

Alternative accounts of priming emphasize the importance of the sensory features shared by the target and the prime context. As suggested by Schmuckler (1989), "a chord sharing component tones, or overtones, with a preceding chord will be more highly anticipated than a continuation containing no overlapping frequencies with its predecessor" (p. 134). In Western music, the potential role of sensory priming is even more crucial because Western rules of harmony are partly correlated with the psychoacoustic structures of sounds (Bigand, Parncutt, \& Lerdahl, 1996; Parncutt, 1989). As a consequence, a target chord and a strongly harmonically related prime chord (e.g., a $\mathrm{C}$ major chord and a $\mathrm{G}$ major chord) generally have more component tones in common than chords that are harmonically less related or unrelated (e.g., a C major chord and an F\# major chord). The strength of harmonic relationship between chords may thus be predicted without invoking music theoretic constructs such as key harmonic hierarchy and psychological constructs or the abstract knowledge of Western musical rules. In the sensory model of Parncutt (1989), harmonic relatedness of two chords is entirely based on the pitch commonality of successive chords, that is, the degree to which the chords have perceived pitches in common, taking into account the relative perceptual salience of each pair of pitches. For example, the pitch commonality value is greater between the chords $\mathrm{C}$ and $\mathrm{G}(.32)$ than between the chords $\mathrm{C}$ and $\mathrm{F} \#(-.12$; for more details, see Bigand et al., 1996; Parncutt, 1989).

In longer musical sequences, the sensory traces associated with every musical event are accumulated in sensory memory. Psychoacoustic models that integrate sensory memory decay may challenge cognitive accounts of Western music perception (see Huron \& Parncutt, 1993; Leman, 1995, 2000; Parncutt \& Bregman, 2000). For example, Huron and Parncutt (1993) provided an alternative account of Krumhansl and Kessler's (1982) key profiles ${ }^{1}$ by integrating sensory memory decay based on pitch salience (see also Leman, 2000). Similarly, a sensory account of harmonic priming would predict that the strength of priming depends on the amount of component tones and virtual pitches the target shares with the component tones and virtual pitches of the prime context stored in sensory memory and decaying over time. This prediction was addressed in Bigand and Pineau (1997) by computing Parncutt's (1989) pitch commonality values between each chord of the prime and the target chord weighted according to recency. Stronger pitch commonality values were observed for tonic target chords than for subdominant target chords, and the pitch commonality values correlated significantly with correct response times. This finding suggests that sensory and cognitive priming may often act in the same direction.

Sensory priming is also related to the frequency of occurrence of the target in the previous context. The more often a target chord occurs in a context, the higher its pitch commonality value with this context. This factor is of great importance for the perception of Western music, because Western tonal and harmonic hierarchies are strongly correlated with the statistical distribution of tones and chords (Krumhansl, 1990a). The most referential musical events (e.g., tonic chords) occur more often than hierarchically less important events (e.g., dominant or subdominant chords). Given that prior presentation of an event enhances its later processing (see Humphreys, Besner, \& Quinlan, 1988; Jacoby, 1983; Jacoby \& Dallas, 1981), several findings in music cognition should be revised with all due attention being paid to the possibility of a repetition priming effect (for a debate, see Butler, 1989, 1990; Krumhansl, 1990b). For example, in Bigand and Pineau (1997), the tonic targets occurred more often in the prime context than did the subdominant targets, suggesting that cognitive and repetition priming may have been partly confounded in this study. Because the most referential events of Western tonal music usually correspond to the most frequent events and to the events that share the most component tones and virtual pitches with the surrounding musical context, differentiating sensory priming from cognitive priming remains a key concern (see Bigand et al., 1996; Bigand \& Pineau, 1997).

A first attempt to investigate this issue was provided by Bigand, Tillmann, Poulin, and Manderlier (2002). For the purpose of the

\footnotetext{
${ }^{1}$ In Krumhansl and Kessler (1982), participants judged how well tones fit in previous tonal contexts. The judgments reflected the differences in tones' functions as defined within the tonal hierarchy. Results have been interpreted in terms of a cognitive representation of tonal relationships by the listener.
} 
experiment, the target chord was a perfect major chord in half of the trials (target trials). In the other half, the last chord was rendered dissonant by adding an additional tone that did not belong to the same key as the chord (foil trials). Participants had to quickly decide whether the last chord was a target or a foil. The critical point was to assess whether consonant-dissonant judgments of the target were affected by a preceding prime chord (for the use of another task in harmonic priming experiments, see Bigand, Tillmann, Poulin, D’Adamo, \& Madurell, 2001; Tillmann $\&$ Bharucha, 2002). In the harmonic priming condition, the target chord was preceded by a harmonically related prime chord (e.g., G major chord $\rightarrow \mathrm{C}$ major chord). In the repetition priming condition, the target chord was identical to the preceding prime chord (e.g., $\mathrm{C}$ major chord $\rightarrow \mathrm{C}$ major chord). The former condition corresponds to the most important harmonic progression of Western music, namely, the authentic cadence. The latter condition features a full overlap in harmonic spectra between prime and target. A cognitive account of harmonic priming predicts stronger priming in the harmonically related condition ( $\mathrm{G}$ major/C major), but a sensory account of priming predicts stronger priming in the repetition condition ( $\mathrm{C}$ major/C major). Results of the consonantdissonant task showed that sensory priming never prevails over cognitive priming: More numerous correct responses and faster correct response times were observed for target chords in the harmonically related condition than for target chords in the repetition priming condition. It is interesting to note that this finding did not depend on the musical expertise of the participants.

The aim of the present study was to analyze sensory and cognitive components of harmonic priming in long contexts. The last chord in eight-chord sequences acted as either a harmonically related tonic chord or a congruent but less harmonically related subdominant chord (see Figure 2). For the purpose of the experiment, the target chord either was a perfect major chord (target trials) or was rendered dissonant by adding a tone played a semitone above one of the chord tones (foil trials). Participants had to quickly decide whether the last chord was a target or a foil. In light of previous research, it was expected that performance in this consonant-dissonant task would be better for tonic target chords than for subdominant target chords (see Bigand et al., 1999; Bigand \& Pineau, 1997; Bigand et al., 2001; Tillmann \& Bigand, 2001; Tillmann et al., 1998). That is to say, more numerous correct responses and faster correct response times in the consonantdissonant task were expected for target chords acting as tonic chords in the prime context.

The critical new point of our study was to contrast cognitive and sensory priming. In the no-target-in-context condition, target chords never occurred in the prime context. The number of notes as well as the number of pitch classes shared by the target and the overall prime context was higher for subdominant targets than for tonic targets (see Tables 1 and 2 and the Appendix). As a consequence, a sensory account of priming predicts greater facilitation for subdominant targets. By contrast, a cognitive account of priming predicts greater facilitation for tonic targets. In the subdominant-in-context condition, the subdominant target (but not the tonic target) occurred in the prime context. Because subdominant targets now shared an even greater number of notes and pitch classes with the prime context, sensory priming should be enhanced, predicting greater facilitation for subdominant targets. Moreover, the difference between the subdominant and tonic tar-
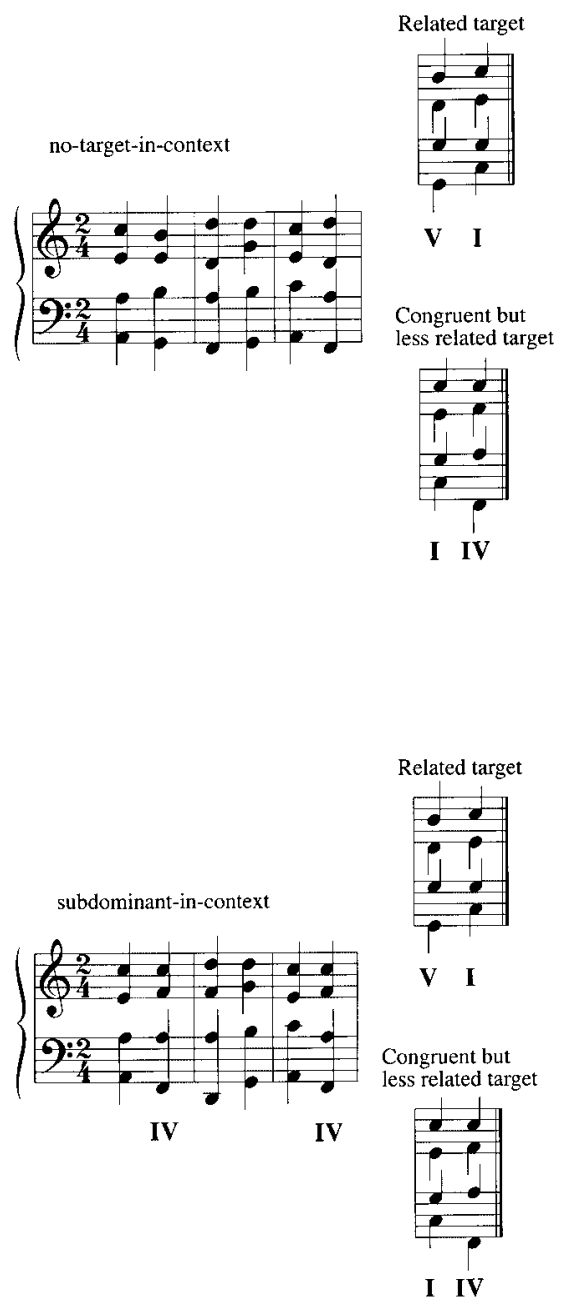

Figure 2. One example of chord sequences used in Experiment 1 in the four experimental conditions. Tonic and subdominant targets are represented by numbers I and IV, respectively (V represents the dominant chord).

gets should be more pronounced in the subdominant-in-context condition than in the no-target-in-context condition. A cognitive account of harmonic priming continues to predict stronger priming for the tonic than the subdominant targets, with no major differences between the subdominant-in-context and the no-target-incontext conditions.

It might be argued that counting the occurrence of each note and pitch class of tonic and subdominant targets in the contexts provides a simplified account of the psychoacoustic differences between the experimental conditions (see Parncutt, 1989; Parncutt \& Bregman, 2000). However, a simple bottom-up stimulus model is strongly correlated with more sophisticated psychoacoustic models that integrate additional parameters, such as pitch salience. For example, Parncutt's pitch commonality values were highly correlated with a simple bottom-up stimulus model in Bigand and Pineau (1997), $r(54)=.92, p<.001$, and in Bigand et al. (1996), $r(48)=.95, p<.001$. Accordingly, more sophisticated psychoacoustic models lead to predictions similar to those of the bottom-up stimulus model. 
Table 1

Averaged Correct Response Times (in Milliseconds) Observed at the Extremely Fast Tempo (75 ms per Chord) as a Function of the Presentation Order of the Tempi and the Harmonic Function of the Target

\begin{tabular}{cccccc}
\hline & \multicolumn{2}{c}{ Nonmusicians } & & \multicolumn{2}{c}{ Musicians } \\
\cline { 2 - 3 } Presentation order & Tonic target & Subdominant target & & Tonic target & Subdominant target \\
\hline Slow fast & $1,023.18(66.67)$ & $1,006.59(60.97)$ & & $696.50(56.20)$ & $740.40(57.42)$ \\
Fast slow & $1,156.45(62.90)$ & $1,004.35(48.20)$ & & $890.26(74.43)$ & $849.44(67.37)$ \\
\hline
\end{tabular}

Note. Standard errors appear in parentheses.

Finally, because sensory and cognitive priming refer to different processes, the present manipulation offers a new opportunity to study the influence of musical expertise. In previous studies, harmonic priming did not vary with musical expertise (Bharucha \& Stoeckig, 1986, 1987; Bigand et al., 1999; Bigand \& Pineau, 1997; Bigand et al., 2001). Nonmusicians' responses were generally less accurate and slower than musicians' responses in the consonant-dissonant judgments, but they reflected similarly the manipulation of the harmonic relatedness of the target. No differences have been reported between the two groups when the perceptual task used in the experiment was as familiar for musicians as for nonmusicians (see Bigand et al., 2001). However, given that sensory and cognitive priming have been confounded in some of the previous priming studies, both components may have been differently active in musicians and nonmusicians, resulting in an overall similar pattern of data for both groups that, however, is based on different processes. The present study allows us to investigate this issue. According to previous studies, we expected stronger cognitive effects than sensory priming effects to be exhibited by musicians, with a reverse tendency for nonmusicians.

\section{Experiment 1}

\section{Method}

Participants. Thirty-five volunteer students participated in this experiment: 19 students of an introductory course at the University of Burgundy, Dijon, France, who had never received formal musical training or learned a musical instrument (referred to below as nonmusicians) and 16 musicol-

Table 2

Number of Pitch Classes Shared by the Targets With All 7 Chords of the Previous Contexts and With the 3 Most Recent Chords, Averaged Over All Experimental Sequences

\begin{tabular}{lccccc}
\hline & \multicolumn{2}{c}{ No target in context } & & \multicolumn{2}{c}{ Subdominant in context } \\
\cline { 2 - 3 } \multicolumn{1}{c}{ Chord } & $\begin{array}{c}\text { Tonic } \\
\text { target }\end{array}$ & $\begin{array}{c}\text { Subdominant } \\
\text { target }\end{array}$ & & $\begin{array}{l}\text { Tonic } \\
\text { target }\end{array}$ & $\begin{array}{c}\text { Subdominant } \\
\text { target }\end{array}$ \\
\hline All 7 chords & 12.50 & 14.75 & & 14.75 & 18.58 \\
Chord 5 & 2.42 & 2.33 & & 2.92 & 3.42 \\
Chord 6 & 0.25 & 3.61 & & 2.25 & 3.75 \\
Chord 7 & 1.50 & 1.92 & & 1.17 & 2.00 \\
\hline
\end{tabular}

Note. A C major chord consisting of the notes (C2-G2-E3-C4), for example, shares one pitch class $(\mathrm{G})$ with a $\mathrm{G}$ major chord consisting of the notes (G1-B2-G3-D4). ogy students at the University of Burgundy who had received formal musical training (the average years of musical training is 12.4 years) or learned a musical instrument (referred to below as musicians). All of the participants received course credit for their participation.

Material. Twelve prime sequences of six chords were used. Each of them ended with a pair of chords that were one step apart on the circle of fifths (e.g., G-C, C-F, or F-Bb). The succession of these two chords forms a local authentic cadence that represents a syntacticlike mark of ending in Western music. The chord sequences thus contained eight chords, all ending on the same type of local harmonic structure (i.e., a local authentic cadence, V-I). The last chord of the sequence defined the target. The harmonic function of this target chord was manipulated so that this last chord functioned either as a stable tonic chord (I) or as a less stable subdominant chord (IV) in the key of the prime context. Figure 2 (top) illustrates this manipulation for 1 of the 12 prime sequence. The displayed prime context is in the $\mathrm{C}$ major key and ended either on the chords $\mathrm{G}$ and $\mathrm{C}$ or on the chords $\mathrm{C}$ and $\mathrm{F}$. In the first ending, the target $\mathrm{C}$ acts as a tonic chord (I). In the second ending, the target $\mathrm{F}$ acts as a subdominant chord (IV). Crossing the 12 prime sequences with the two types of endings resulted in 24 chord sequences.

Given that the harmonic function of the target chord was manipulated by changing the last two chords of the musical sequences, a further control was performed within the total set of chord sequences. Each chord pair containing the target was used to define both the tonic condition and the subdominant condition. For example, the chord pair G-C of Figure 2 (top) ended a different prime sequence in the key of $\mathrm{G}$ major, and the target $\mathrm{C}$ now acted as a subdominant chord. Similarly, the pair C-F (Figure 2, top) was used with another prime sequence in the key of $\mathrm{F}$ major, and the target $\mathrm{F}$ now acted as a tonic chord. Accordingly, over the whole set of chord sequences, the manipulation of the harmonic function of the target chord was not confounded with changes in chords that defined the targets.

In addition to manipulating the harmonic function of the target, we manipulated the occurrence of the subdominant chord in the prime context, resulting in $48(24 \times 2)$ experimental chord sequences. In the no-targetin-context condition, the targets (tonic and subdominant) never occurred in the prime context. In the subdominant-in-context condition, the subdominant chord occurred once or twice in the context but not the tonic chord (Figure 2, bottom). Subdominant targets shared numerous notes and pitch classes with the prime context in all conditions (see Tables 1 and 2 and the Appendix). Moreover, the difference between tonic and subdominant targets concerning both notes and pitch classes was generally more pronounced for the most recent chords of the prime.

For the purpose of the experiment, the last chord was consonant (target chord) in half of the trials and was rendered dissonant in the other half (foil trials). The dissonance was created by adding a tone that was one semitone above the tonic or the fifth of the target chord (e.g., either the tone $\mathrm{C \#}$ or $\mathrm{G} \#$ was added to the $\mathrm{C}$ chord consisting of the tones $\mathrm{C}-\mathrm{E}-\mathrm{G})$. This added tone was rendered less salient by decreasing its amplitude by $4 \mathrm{~dB}(37.5 \%)$. 
Apparatus. The stimuli were played with sampled piano sounds produced by an ETM10 Yamaha Sound Expander (Yamaha Corporation, Shizuoka, Japan). The first seven chords of the sequences were played at a tempo of 96 quarter notes per minute (660 ms per chord). Stimulus onset asynchrony (SOA) was $660 \mathrm{~ms}$. The eighth chord of the sequence was played for 2,000 ms. The Yamaha sampler was controlled by the MIDI interface of a Macintosh computer running Performer software (Mark of the Unicorn, Cambridge, MA). The sound stimuli were captured by SoundEditPro software (Macromedia, Inc., San Francisco, CA) at CD quality (16 bits and $44.1 \mathrm{kHz}$ ). The experiment was run with Psyscope software (Cohen, MacWhinney, Flatt, \& Provost, 1993).

Procedure. The experimental procedure was split into two phases. In the first phase, participants were trained to differentiate between targets and foils with 16 chords played in isolation and in random order. They were asked to decide as quickly and as accurately as possible whether the target chord is acoustically consonant (target) or dissonant (foils). They were informed by a feedback sound signal when their response was incorrect. In the second phase, participants performed this consonantdissonant judgment for the last chord of each sequence. A feedback signal sounded following incorrect responses. After each chord sequence, an interfering sequence of randomly selected pure tones was played.

Design. Crossing the harmonic functions of the target (tonic vs. subdominant) and the occurrence of the subdominant (no target in context vs. subdominant in context) resulted in 48 chord sequences. This experimental material was split into two sets of 24 chord sequences (Set 1 and Set 2). For half of the participants, Set 1 was presented with targets and Set 2 was presented with foils. For the other half of the participants, the attribution of chord type (target vs. foil) to the two sequence sets was reversed. Each participant thus heard 24 sequences ending on a target and 24 sequences ending on a foil. The chord sequences were transposed in the 12 major keys, with four chord sequences per major key. The musical expertise (musicians vs. nonmusicians) defined the between-subjects variable. The harmonic function of the target chord (tonic vs. subdominant) and the occurrence of the subdominant in the chord sequence defined the withinsubject variables. None of these variables were blocked.

\section{Results}

As foils do not correspond to lawful musical events in Western tonal music, no straightforward prediction can be made for the foil trials. ${ }^{2}$ Foil trials were used as fillers, and only the data of target trials are presented below. Percentages of correct responses are displayed in Figure 3 (top). Arcsine transforms of these percentages were analyzed with a 2 (musical expertise) $\times 2$ (harmonic function) $\times 2$ (occurrence of the subdominant) analysis of variance (ANOVA). Correct responses were more numerous for tonic targets than for subdominant targets, $F(1,33)=8.04, p<.01$, $M S E=169.49$. The effect of the harmonic function of the target was stronger in nonmusicians, as revealed by a Harmonic Function $\times$ Musical Expertise interaction, $F(1,33)=4.53, p<.05$, $M S E=169.49$. Correct responses were more numerous for musicians than for nonmusicians, $F(1,33)=9.23, p<.01, M S E=$ 409.39. There were no other significant effects.

Correct response times are displayed in Figure 3 (bottom). Correct responses were faster for tonic targets than for subdominant targets, $F(1,33)=38.12, p<.001, M S E=17,256.20$, and this effect was stronger for nonmusicians, as shown by a significant interaction between musical expertise and harmonic function, $F(1,33)=5.38, p<.05, M S E=17,256.20$. Musicians were faster than nonmusicians, $F(1,33)=8.26, p<.01, M S E=198,107.40$. There were no further significant effects. It is interesting that averaged correct response times were almost identical in the no-
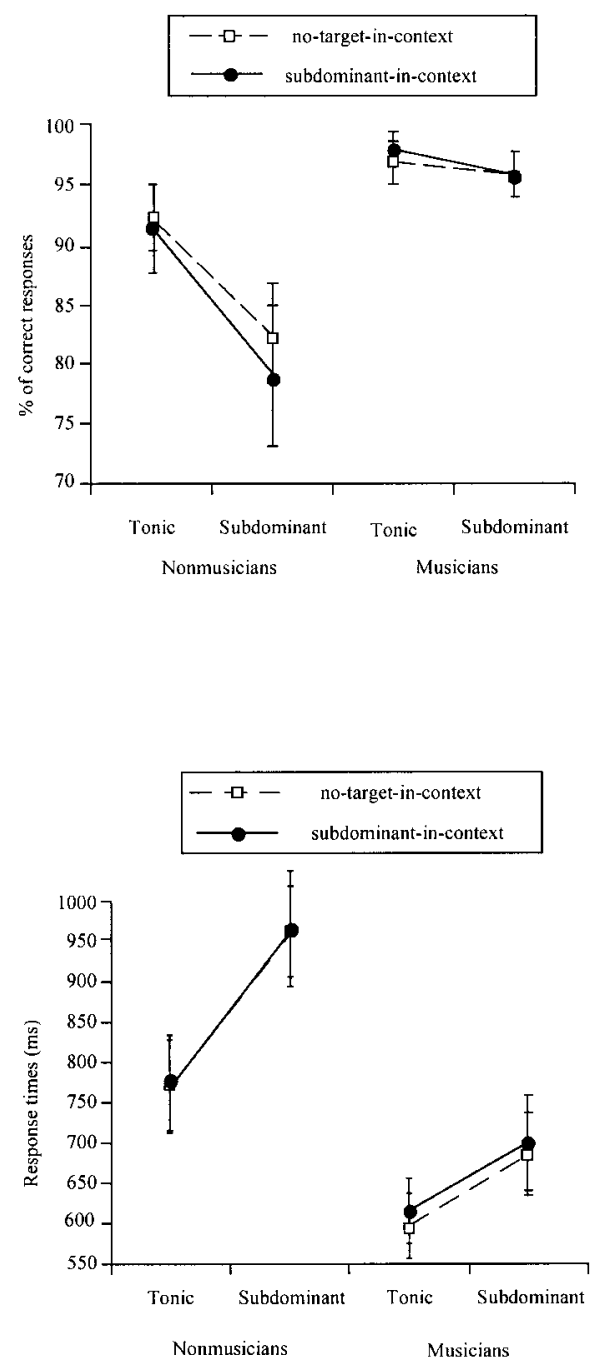

Figure 3. Correct response rates (top) and correct response times (bottom) observed in Experiment 1 for both groups of participants and for tonic and subdominant targets. Error bars represent standard errors.

target-in-context condition and the subdominant-in-context condition.

To assess whether the facilitation observed for tonic target chords may have resulted from an increasing familiarity with the chord sequences during the course of the experiment, we split the experimental trials into two blocks. The first block was made of the first 24 trials, the second block of the last 24 trials (determined individually for each participant). An ANOVA, with block (2) as

\footnotetext{
${ }^{2}$ In the published literature on harmonic priming, the data of the foil conditions were generally not consistent, with some experiments showing crossover interaction between chord types (consonant vs. dissonant) and harmonic context. By contrast, the data of the musically valid target conditions were strongly consistent over all experiments. This difference in consistency of the data suggests that responses to foils involve different cognitive processes than do responses to targets. Consequently, combining data of both chord types in the same analysis might be considered as problematic.
} 
an additional variable, mirrored the outcome of the previous analysis. Averaged correct response times exhibited identical patterns for the first and second block of trials. In each block, correct responses were faster for tonic targets than for subdominant targets, $F(1,28)=35.77, p<.001, M S E=28,850.70$. Not surprisingly, correct responses were faster for the second half of trials, $F(1,28)=21.48, p<.001, M S E=14,786.40$, but there was no significant interaction involving block.

\section{Discussion}

Experiment 1 replicated Bigand and Pineau's (1997) finding with an experimental setting that manipulates the potential role played by sensory priming. Target chords were more correctly and more quickly processed when they act as tonic rather than subdominant chords, even though the targets never occurred in the prime context and the tonic chords shared fewer notes and pitch classes with the prime context (no-target-in-context condition). This outcome confirms that harmonic priming in chord sequences reported in previous studies was unlikely to be caused only by sensory priming. The critical point of Experiment 1 was to demonstrate that cognitive priming continued to prevail over sensory priming even when the frequency of occurrence of the less related targets in the context was increased. In the subdominant-in-context condition, the subdominant targets occurred in the prime context, contrary to the tonic targets that did not. The subdominant targets shared more notes and pitch classes with the prime context than did the tonic targets. According to a sensory account of priming, greater facilitation should have been observed for the subdominant targets. However, stronger priming continued to be observed for tonic targets, and there was no noticeable decrease in the size of the facilitation effect between the no-target-in-context condition and the subdominant-in-context condition. Cognitive priming prevailed over sensory priming from the beginning of the experimental trials, suggesting that the strength of the cognitive component does not depend on the familiarity with musical stimuli.

Experiment 1 demonstrated that the processing of a target chord is mostly determined by its harmonic function in the context and only weakly, if any, by the sensory features the chord shares with the previous musical events. This finding emphasizes the strength of a cognitive component but does not rule out the potential role of a sensory component in musical priming. In a previous study, Tekman and Bharucha (1998, Experiment 1) showed that sensory priming overrules cognitive priming for an SOA of $50 \mathrm{~ms}$ but that cognitive priming prevails for SOAs of 500, 1,500, or 2,500 ms. This finding suggests that sensory and cognitive priming differ in their time course, with top-down influences needing more time than bottom-up influences. The moderate tempo used in Experiment 1 (660 ms per chord) may explain the strength of the cognitive component over the sensory component. At this tempo, listeners may have had enough time to infer the key of the sequence and to prime the processing of the tonic chord. The purpose of Experiment 2 was to investigate the course of the sensory and cognitive components by speeding up the tempo of the prime context ( $300 \mathrm{~ms}, 150 \mathrm{~ms}$, and $75 \mathrm{~ms}$ per chord). Cognitive priming was expected to prevail over sensory priming at moderately fast tempo, and this difference was expected to reverse as the tempo increases, with stronger sensory priming at an extremely fast tempo. In addition, varying the tempo of the prime chords offered a further opportunity to investigate the influence of musical expertise. The speed at which both sensory and cognitive components take place was likely to differ with the extent of musical expertise. A long training and a sustained practice in music may speed up the functioning of the cognitive component in musicians. Accordingly, cognitive priming was expected to be still active at very fast tempi in musicians and to be less influential than sensory priming in nonmusicians.

\section{Experiment 2}

\section{Method}

Participants. Forty volunteer students participated in this experiment: 19 students of an introductory course at the University of Burgundy who had never received formal musical training or learned a musical instrument (referred to below as nonmusicians); 21 students from the Music Conservatories of Dijon and Grenoble, France, who had received formal musical training, learned a musical instrument for 10 years on average, and were qualified as candidates for the final diploma of the Conservatory (referred to below as musicians). All of the participants received course credit or were paid \$7. None had participated in Experiment 1.

Material and apparatus. Material and apparatus were as described in Experiment 1, except for the tempo of the chord sequences. The tempo varied from moderately fast (300 ms per chord) to fast (150 ms per chord) to extremely fast ( $75 \mathrm{~ms}$ per chord). The duration of the last chord-target or foil-was set at $2,000 \mathrm{~ms}$. The interchord interval was set at 0 . The chord sequences were transposed in the 12 major keys, with eight chord sequences per major key.

Procedure and design. The procedure was as described in Experiment 1. Crossing the manipulations of the harmonic function of the target (tonic vs. subdominant), the occurrence of the subdominant (no target in context vs. subdominant in context), and the tempo of the prime $(300,150$, and $75 \mathrm{~ms}$ per chord) resulted in 12 experimental conditions. Each participant heard 96 sequences (48 presented with a target, 48 with a foil) separated in three blocks as a function of the tempo of the prime. Half of the participants started with the extremely fast tempo, followed by the fast tempo, and then by the moderately fast tempo blocks. This presentation order was reversed for the other half of the participants. Before each block, participants were informed that the chord sequences would be played either more rapidly or more slowly. The musical expertise (musicians vs. nonmusicians) defined the between-subjects variable. The harmonic function of the target chord (tonic vs. subdominant) and the occurrence of the subdominant in the chord sequence defined the within-subject variables. None of these variables were blocked.

\section{Results}

Figure 4 (top) displays the percentages of correct responses for the target trials. The arcsine transforms of these percentages were analyzed with a 2 (musical expertise) $\times 2$ (harmonic function) $\times$ 2 (occurrence of the subdominant) $\times 3$ (tempo of the prime) ANOVA. There was a main effect of the harmonic function of the target, with more correct responses for tonic targets than for subdominant targets, $F(1,38)=12.67, p<.01, M S E=385.22$; a main effect of tempo, with fewer correct responses for the fast tempo (150 ms per chord), $F(2,76)=3.36, p<.05, M S E=$ 459.69; and a main effect of musical expertise, with fewer correct responses for nonmusicians, $F(1,38)=31.66, p<.001, M S E=$ 1,808.52. The epsilon values (.897) did not result in significant 
$--\square-$ no-target-in-context

$\longrightarrow$ subdominant-in-contex

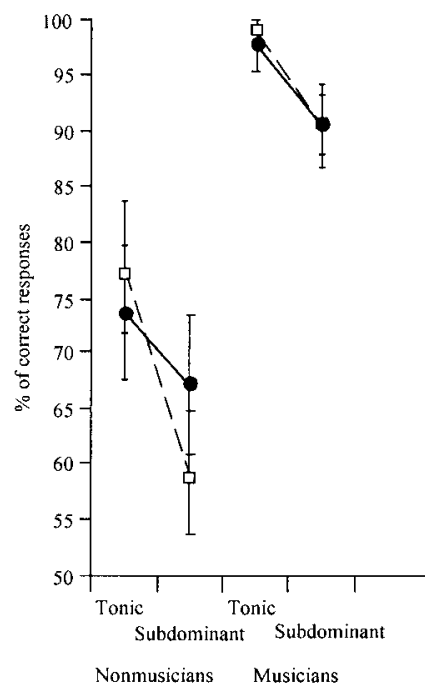

$300 \mathrm{~ms}$
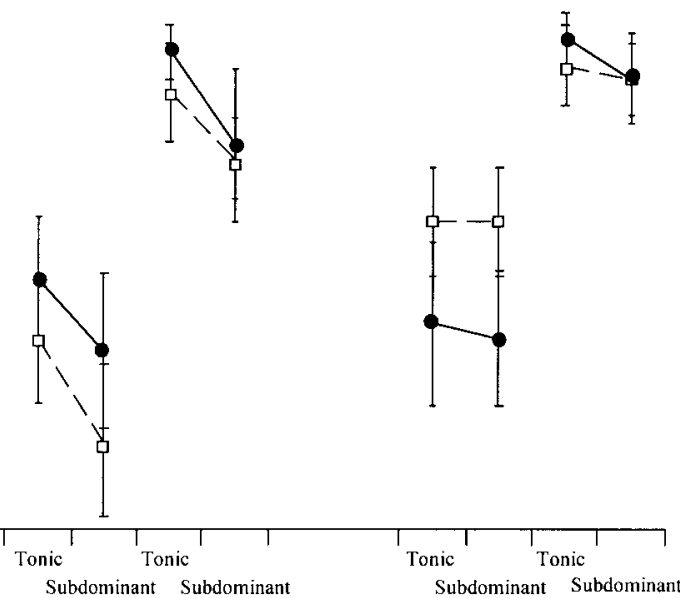

Nonmusicians Musicians
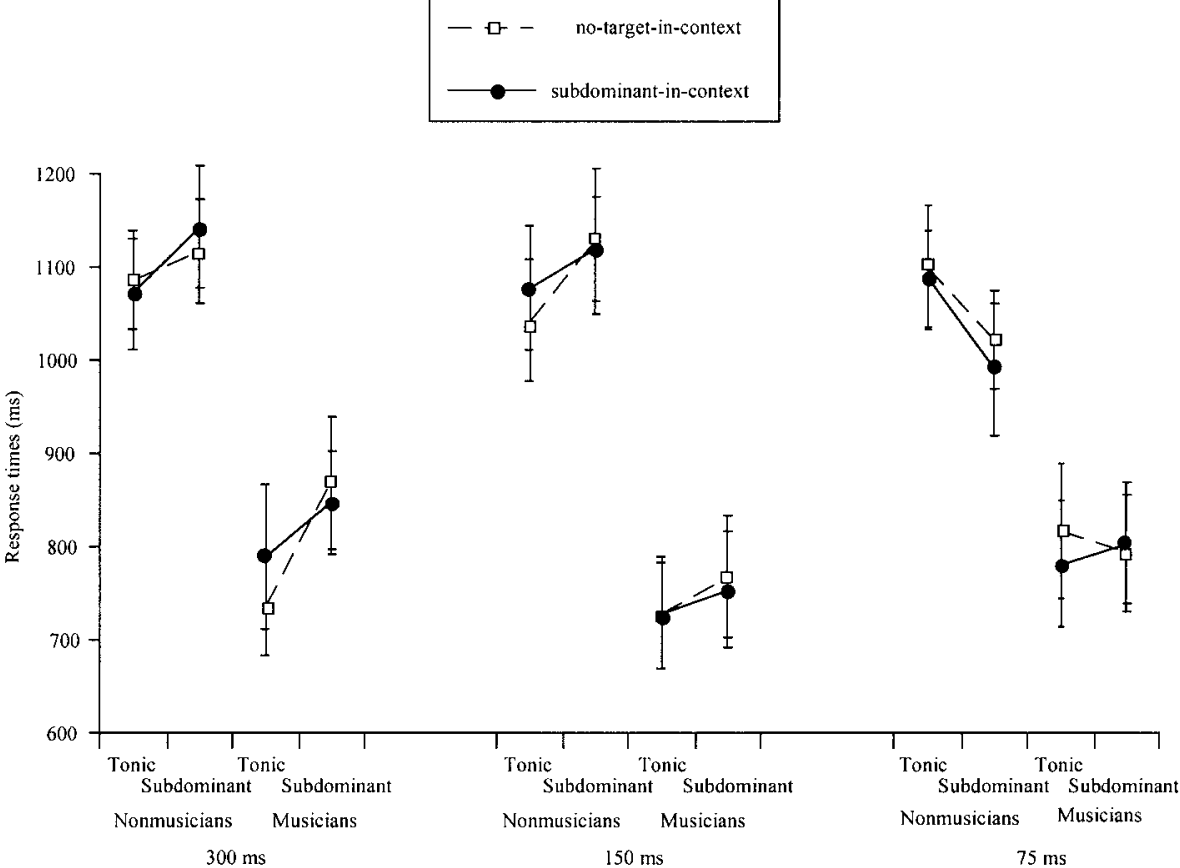

Figure 4. Correct response rates (top) and correct response times (bottom) observed in Experiment 2 for both groups of participants and for tonic and subdominant targets. Error bars represent standard errors.

changes in $F$ values after Greenhouse-Geisser's correction. Contrast analyses indicated that the effect of harmonic function of the target decreased as the tempo of the prime increased, $F(1,38)=$ 3.94, $p<.05, M S E=14,225.80$. As expected, responses were more accurate for the tonic targets than subdominant targets at a moderately fast tempo, $F(1,38)=13.25, p<.001, M S E=$ $11,711.31$, and, to a lesser extent, at a fast tempo, $F(1,38)=4.44$, $p<.05, M S E=19,306.86$. At an extremely fast tempo, there was 
no effect of the harmonic function of the target, $F(1,38)<1$. There were no other significant effects. ${ }^{3}$

Correct response times mirrored the global pattern of correct response rates (see Figure 4, bottom). A $2 \times 2 \times 3$ ANOVA on response times showed a significant interaction between harmonic function and tempo, $F(2,76)=8.11, p<.001, M S E=18,857.80$. The epsilon values (.994) did not result in significant changes in $F^{\prime}$ values after Greenhouse-Geisser's correction. Contrast analyses indicated that priming reversed with increasing tempo of the context, $F(1,38)=11.90, p<.001, M S E=878,764.10$. Responses were faster for tonic targets than for subdominant targets at the moderately fast tempo, $F(1,38)=8.03, p<.001, M S E=$ $1,013,793.00$, and at the fast tempo, $F(1,38)=8.30, p<.001$, $M S E=430,428.10$, but not at the extremely fast tempo. For the extremely fast tempo, response times were marginally shorter for the subdominant targets than for the tonic targets, $F(1,38)=3.88$, $p<.06, M S E=791,926.70$, and this effect reached a statistically significant level in nonmusicians only, $F(1,38)=7.32, p<.01$, $M S E=761,926.70$. Further contrasts revealed that response times for tonic targets did not significantly vary with the tempo, $F(1$, $38)<1$, and only response times for subdominant targets decreased as the tempo increased, $F(1,38)=7.81, p<.01, M S E=$ $1,603,965.00$. There were no further significant effects, except that musicians responded faster than did nonmusicians, $F(1,38)=$ $15.10, p<.001, M S E=702,754.00$.

Given that the extremely fast tempo was presented at the beginning of the experimental session for half of the participants and at the end of the experimental session for the other half of the participants, it was of interest to analyze whether the presentation order of tempi may have influenced the data. The results of an ANOVA including the additional variable, presentation order of tempi, mirrored the previously presented outcome but pointed out a marginally significant three-way interaction of Harmonic Function $\times$ Tempo $\times$ Presentation Order of Tempi, $F(2,72)=2.87$, $p=.062, M S E=17,814.80$. This interaction was caused by the extremely fast tempo. Irrespective of the presentation order of tempi, correct responses were faster for tonic targets than subdominant targets for both moderately (300 ms) and fast tempi (150 ms), whereas correct responses were faster for subdominant targets at the extremely fast tempo $(75 \mathrm{~ms})$. The critical finding is that this advantage of the subdominant targets was more pronounced for participants who began the experimental session with the extremely fast tempo, $F(1,36)=6.81, p<.01, M S E=17,704.10$ (see Table 1), and this advantage did not reach significance in the other group of participants who ended the experimental session with the extremely fast tempo. ${ }^{4}$ There was no further significant interaction.

Sensory priming thus predominated over cognitive priming at extremely fast tempo only for participants who did not encounter the chord sequences at slower tempi previously in the experimental session. Another way to describe this interaction is to state that tonic target chord processing took greater advantage from previous listening of chord sequences at slower tempi than did subdominant target chord processing. Combined with the fact that cognitive priming clearly predominated at slower tempo, the present finding indicates that the extremely fast tempo $(75 \mathrm{~ms})$ was probably too fast to activate cognitive priming processes but not fast enough to hinder or remove a cognitive-listening-processing mode that has been already well established in listeners by slower chord sequences. This conclusion is valid for both musicians and nonmusicians, even though nonmusicians seemed to be overall slightly more sensitive to sensory priming at this extremely fast tempo.

A possible alternative account of the finding observed at $75 \mathrm{~ms}$ would argue that the repeated chord creates a new stream at extremely fast tempo. This new stream would make the repeated chord more salient, which would result in easier processing at 75 $\mathrm{ms}$ for subdominant targets. Stream segregation is, however, unlikely to occur in the present chord sequences. Stream segregation involves a discontinuity in sound features, which is not present in our chord sequences (for a complete review, see Bregman, 1990). Moreover, Bregman demonstrated that stream segregation through repetition is a cumulative process that takes time to occur. Rather than immediately breaking a sequence into streams as soon as a few tones have fallen in different frequency ranges, the auditory system waits and only gradually increases the tendency for the streams to segregate as more and more evidence builds up to favor a two-source interpretation (see Bregman's, 1990, CD demo 4 and Bregman, 1990, pp. 128-130). The weak number of evidence for segregation (two to three repetitions) as well as the extremely short duration of the chord sequence in the 75-ms condition cannot result in stream segregation.

\section{Discussion}

In comparison with Experiment 1, the tempo of the prime context was considerably increased in Experiment 2: The slowest tempo (300 ms per chord) was over twice as fast, and the fastest tempo was over six times faster ( $75 \mathrm{~ms}$ per chord). This increased tempo of the prime context resulted in fewer correct responses and longer correct response times for musicians and nonmusicians. Nevertheless, for two of the three tempi, Experiment 2 replicated Experiment 1. For the tempi of 300 and $150 \mathrm{~ms}$ per chord, cognitive priming prevailed over sensory priming, and this result was unaffected by the repetition of the subdominant chord in the context. In addition, the present finding points out that the inference of the key underlying a musical sequence and the inference of the harmonic function of a chord both occur in a very fast way.

This outcome converges with a recent event-related potential study: The difference in harmonic functions of the tonic and subdominant targets was associated with a difference in positivity peaking very early at $300 \mathrm{~ms}$ after target onset (Regnault, Bigand, $\&$ Besson, 2001). With the stimuli of the present study, the fast inference of a major key is rather surprising. The chord sequences consisted mostly of minor chords, and two of the most referential major chords (including the most referential tonic) only occurred as the target chord at the very end of the sequence (no-target-in-

\footnotetext{
${ }^{3}$ The difference in accuracy responses observed in nonmusicians for the subdominant targets between the subdominant-in-context and the notarget-in-context conditions did not reach significance at $300 \mathrm{~ms}(p>.14)$ or at $150 \mathrm{~ms}(p>.23)$.

${ }^{4}$ The slight difference in average correct response times observed in the slow-fast groups of nonmusicians $(1,023.18$ vs. 1,006.59) did not reach statistical significance.
} 
context condition). The fact that the result pattern of nonmusicians did not differ from the result pattern of musicians highlights the sophistication of the musical ability of musically naive listeners. The only difference between the two groups concerned their performance accuracies in the consonant-dissonant judgments: Correct responses were more numerous and were faster in musicians. This result is not surprising because the experimental task taps into a perceptual competence that is explicitly trained in music education classes. When the perceptual task is as familiar for musicians as for nonmusicians, no statistically significant differences in response times were reported between these groups (see Bigand et al., 2001). This outcome suggests that harmonic priming involves a very robust cognitive component that does not need explicit training.

The crucial finding of Experiment 2 was that the difference between tonic and subdominant targets changed as the tempo increased from $300 \mathrm{~ms}$ to $75 \mathrm{~ms}$. Whereas tonic targets continued to be processed similarly with increasing tempo, subdominant targets took advantage from the increased tempo. Given that subdominant targets shared more notes and pitch classes with the prime context, it is likely that sensory priming became more and more powerful as the tempo increased. At extremely fast tempo, the strength of sensory priming effects depended on the temporal context of the experiment. Sensory priming prevailed over cognitive priming only for participants who had not listened to chord sequences at slower tempi beforehand. For those who had already practiced the task with chord sequences played at slower tempi, cognitive priming processes remained influential and were roughly as influential as sensory priming processes. Taken together, the data provided some evidence that sensory priming effects observed at extremely fast tempi are rapidly overruled by cognitive priming effects when the tempo decreases. By comparison, processes underlying cognitive priming need more time than $75 \mathrm{~ms}$ per chord to be activated, but, once activated, they remain influential even at this extremely fast tempo.

\section{General Discussion}

The role played by knowledge-driven processes in Western harmony perception has been questioned by several authors (Butler, 1989; Huron \& Parncutt, 1993; Leman, 1995, 2000; Parncutt \& Bregman, 2000). It was argued that some data usually referred to as support for the cognitive foundation of music perception do not necessarily reflect an abstract knowledge of Western music rules but may be more simply accounted for by psychoacoustic models of short-term memory. For example, Krumhansl and Kessler's (1982) key profiles emerged from an echoic memory model based on pitch salience (Huron \& Parncutt, 1993; see also Parncutt \& Bregman, 2000) and from a short-term memory model based on echoic images of periodicity pitch (Leman, 2000).

The present study supports a rather different view: Knowledgedriven processes prevail in Western harmony perception even at tempi as fast as $300 \mathrm{~ms}$ and $150 \mathrm{~ms}$ per chord. In both experiments, tonic target chords that act as the most referential chord of Western harmony but share the fewer pitch classes with the context were the most strongly primed. In contrast to predictions of psychoacoustic models that integrate sensory memory decay, the subdominant targets sharing numerous identical notes as well as numerous pitch classes with the preceding context chords were less primed. More challenging for a sensory account of harmonic processing was the finding that increasing the overlap in pitch classes and identical notes between the less related subdominant targets and the prime context (by allowing the occurrence of the subdominant chord in the prime context) never affected the strength of harmonic priming. These findings provide converging evidence that anticipatory processes intervening during music listening essentially occur at a cognitive level of representation. They are consistent with previous experiments investigating these processes (see Bharucha \& Stoeckig, 1987; Bigand \& Pineau, 1997; Tekman \& Bharucha, 1992).

The single evidence for a contribution of sensory-driven processes was reported in Experiment 2 when the tempo of the prime was increased to an extremely fast tempo. However, it is striking that the effects of sensory priming overruled those of cognitive priming only for participants who had never heard the chord sequences played at a slower tempo. The influence of sensorydriven processes at extremely fast tempo is consistent with the finding reported by Tekman and Bharucha (1998) in two-chord priming experiments. The authors showed that cognitive priming needs more time than sensory priming, with cognitive priming prevailing over sensory priming at very slow tempi and sensory priming prevailing over cognitive priming at extremely fast tempi. The present study adds new evidence about the course of sensory and cognitive priming by showing that, once activated, the cognitive process is an extremely fast-acting component that can compete with sensory priming, even at extremely fast tempi. In addition, it should be emphasized that a tempo of $75 \mathrm{~ms}$ per sound is considerably faster than tempi usually encountered in music. ${ }^{5}$ As noted by Fraisse (1967), the fastest rate normally used for melodic themes is about $150 \mathrm{~ms}$ per note. In other words, for most of the tempi found in everyday life for Western music, cognitive priming will prevail over sensory priming in large contexts.

Bharucha's (1987) connectionist model of Western tonal knowledge representation proposes an elegant account of the cognitive component of priming. In this model (referred to as MUSACT), knowledge of the Western harmonic hierarchy is conceived of as a network of interconnected units. These units are organized in three layers, which correspond to tones, chords, and keys. Each of the 12 tones is connected to six chord units representing the three major chords and the three minor chords of which that tone is a component. In the same way, each chord unit is connected to three major key units representing the keys of which that chord is a component. This pattern of connections constitutes a knowledge representation of Western harmony that generates automatic and schematic expectations (Bharucha, 1987, 1994).

When a triad (i.e., a chord consisting of three tones) is sounded, the three corresponding tone units are activated, and phasic activation (i.e., change of activation) spreads through connections toward chord units. The chord unit connected to all three tones

\footnotetext{
5 A tempo of $75 \mathrm{~ms}$ per chord is close to the threshold for the identification of the order of tones (125 ms for pure tones) and of vowels (100 ms; Thomas \& Fitzgibbons, 1971) and below the threshold for the recognition of well-known melodies (150 ms on average; see Warren, 1993).
} 


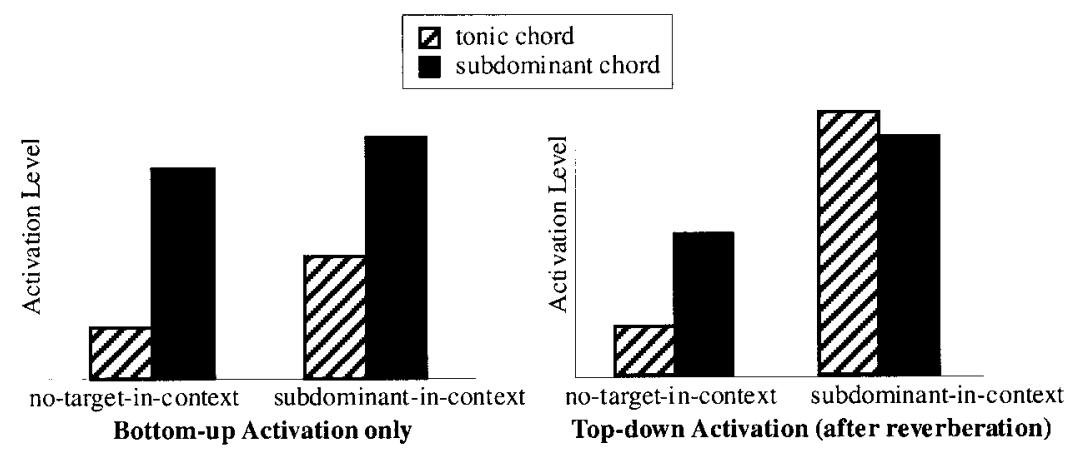

Figure 5. Activation of the target chord units (representing the tonic and the subdominant chords) with bottom-up activation only (left) and with top-down activation after reverberation (right) in the two experimental conditions.

receives the strongest activation. For example, if the triad includes the tones $\mathrm{C}, \mathrm{E}$, and $\mathrm{G}$, the $\mathrm{C}$ major chord is the most activated chord unit. Phasic activation spreads from chord to key units (bottom-up activation) and reverberates toward tone units (topdown activation) and back to chord and key units, and so on. After several cycles of reverberation, MUSACT reaches equilibrium (i.e., reverberating phasic activation is inferior to a given threshold), and the pattern of activation reflects tonal and harmonic hierarchies. In long chord sequences, activations due to each chord are accumulated, and their pattern of activation decays over time (according to recency). The global pattern of activation in the units at the end of the sequence represents the influence of the overall context. The activation levels are interpreted as levels of expectation for subsequent events (Bharucha, 1987; Bharucha \& Stoeckig, 1987; Bigand et al., 1999; Tekman \& Bharucha, 1998; Tillmann et al., 1998) and predict harmonic priming: After the presentation of the prime context, the activation of a target chord unit is stronger for a tonic chord than for a subdominant chord (Bigand et al., 1999).

Harmonic priming studies to date have provided strong support for this model, either with short contexts (Bharucha \& Stoeckig, 1987; Tekman \& Bharucha, 1998; Tillmann \& Bharucha, 2002) or with long contexts (Bigand et al., 1999; Tillmann \& Bigand, 2001; Tillmann et al., 1998). Our present study provides a challenging test for the model that simulates the time course of bottom-up and top-down activation in short prime contexts (see Tekman \& Bharucha, 1998). The chord sequences and their different tempi of presentation were designed to contrast sensory priming and cognitive priming. The connectionist model should lead to different predictions depending on whether only the bottom-up activation is considered or whether top-down reverberation is included. In other words, bottom-up activation should result in stronger activation for subdominant targets, and top-down activation should result in stronger activation for tonic targets.

Simulations were performed with the chord sequences used in Experiment 1. The first seven chords were presented one by one to the model, and the activation of the target chord units was read off after the seventh chord. The accumulated activation pattern was based either on bottom-up activation only (the system did not reverberate yet) or on activation after reverberation at the state of equilibrium (including top-down influences). ${ }^{6}$ As shown in Figure
5, bottom-up activation correctly simulated strong sensory priming. After reverberation, top-down activation wrongly simulated stronger priming for subdominant chords in the no-target-incontext condition but correctly simulated slightly stronger priming effects for tonic chords in the subdominant-in-context condition. This last finding was far from obvious, given that the less activated subdominant target did frequently occur in the context, and it suggests that MUSACT simulates some aspects of the power of cognitive priming. We thus may wonder why MUSACT predicted the opposite direction in the no-target-in-context condition with stronger activation of the subdominant chord even after reverberation. In this condition, chord sequences mostly contained minor chords. Given that both tonic and subdominant chords were avoided, musical substitutions of these chords were used (the minor third and the sixth degree for the tonic and the minor second degree for the subdominant). The present finding suggests that Western listeners, in contrast to the MUSACT model, have some mental representation of these substitutions.

The initial model of MUSACT (Bharucha, 1987) did not include a representation of the functional equivalences between major and minor chords. These functional equivalences may be, ideally, instantiated by horizontal connections between major and minor chords. Horizontal connections are frequent in interactiveactivation models simulating word recognition (McClelland \& Rumelhart, 1981; Rumelhart \& McClelland, 1982) and speech recognition (Elman \& McClelland, 1984; McClelland \& Elman, 1986). The main question would be to explain how MUSACT may learn functional equivalences. In the competitive learning algorithm used by Tillmann et al. (2000), the neural net becomes gradually sensitive to statistical regularities encountered in the

\footnotetext{
${ }^{6}$ For top-down activation, activation after each chord was reverberating until equilibrium. Fifty-four cycles (i.e., the definition of a cycle is based on one update of activation in all three layers) were necessary to reach equilibrium in the implementation of MUSACT in Matlab (Bigand et al., 1999). For bottom-up activation, just 1 cycle was allowed: The input layer sent activation to the chord layer, which then sent it to the key layer. The activation of chord units was thus determined only by the incoming activation from the tone layer.
} 
musical chords and sequences presented to the model. Before training, the units of the first and second layers of the hierarchical self-organizing map (SOM) were all interconnected, as well as those of the second and third layer. During training, the units of the second layer became specialized in the detection of major and minor chords, and those of the third layer became specialized in the detection of keys. However, the SOM did not contain connections between units inside a layer. To allow the neural net to learn functional equivalence between major and minor chords, one would need to include horizontal connections inside a layer that are modified during the training session. Future research should be devoted to address this issue.

\section{Conclusion}

Taken in combination with several other studies on harmonic priming, the present finding suggests that sensory components play a relatively minor role in harmonic priming. This finding has several theoretical implications. First, it emphasizes the relative autonomy of the cognitive component involved in music perception for both musically naive and expert listeners. This conclusion does not mean that psychoacoustic models do not capture relevant features for music perception. The Western musical system is likely to have been originally influenced by the acoustical structure of sounds and by the constraints imposed by the auditory system. Through history, cultural conventions led the musical system to shed these initial acoustical and sensory constraints (see Bharucha, 1994). It is likely that current psychoacoustic models capture some residual traces of these initial influences on the Western musical system. However, the question arises concerning the extent to which a contemporary Western listener has a mental representation of the complex historical entwining between cultural conventions and acoustical features of sounds. It seems more parsimonious to represent mentally only the final state of this historical evolution of the musical system.

In a related vein, the finding that sensory priming rarely prevails over cognitive priming does not imply that the statistical distributions of tones or chords in Western music are not psychologically relevant. The frequency of occurrence of events and of event combinations is likely to contribute to the internalization of the Western harmonic hierarchy (see Tillmann et al., 2000). However, once the musical system has been internalized, it seems no longer necessary that musical pieces follow the usual statistical regularities to be perceived with their underlying harmonic hierarchy. Knowledge-driven processes contribute to process the musical functions of events, even though these functions are not emphasized by the frequency of occurrence in the context. The present findings thus do not refute the importance of sensory processes or of statistical distribution, but they emphasize the relative autonomy of the cognitive component involved in music perception once learning has occurred. One may wonder whether the strength of the cognitive component, as revealed by the present study, can be generalized to other harmonic functions than the tonic and the subdominant. Preliminary results obtained with the resolutions of the diminished seventh chord confirmed that cognitive priming continues to prevail over sensory priming even for less structurally important harmonic functions (Poulin, Bigand, D’Adamo, Madurell, \& Tillmann, 2000).
A second theoretical implication concerns the apparent weakness of repetition priming in the music domain. It has been well established that the processing of a target event is usually facilitated if this event has been presented prior to the target. An event perceived at one point in time generally facilitates the perception of that same event later in time. ${ }^{7}$ This repetition priming effect is robust and has been demonstrated at both behavioral and electrophysiological levels (Besson \& Kutas, 1993; Radeau, Besson, Fonteneau, \& Castro, 1998) and in a variety of experimental paradigms, such as lexical decision task (Forster \& Davis, 1984), word identification (Jacoby \& Witherspoon, 1982), completion of degraded versions of target events (Humphreys et al., 1988; Tulving, Schacter, \& Stark, 1982), determination of recognition thresholds (Feustel, Shiffrin, \& Salasoo, 1983; Jacoby, 1983), and use of different types of events, such as environmental sounds (Chiu \& Schacter, 1995; Stuart \& Jones, 1995) and drawings and pictures of common objects (Srinivas, 1993). The present study, along with Bigand et al. (2002), is among the first ones in the music domain to challenge the strength of repetition priming. Future research has to investigate whether this weakness of repetition priming is specific to musical stimuli or may be reproduced with other types of stimuli.

A further theoretical implication of the present study concerns the weak influence of musical expertise. Recent research in neuroimagery has emphasized the differences between the brains of musically expert and novice listeners, suggesting that both groups of listeners may perceive music rather differently (Ohnishi et al., 2001; Pantev, Hoke, Lütkenhöner, \& Lehnertz, 1989; Pantev \& Lütkenhöner, 2000). The present study provides challenging evidence for this claim. Beyond main differences in accuracy responses and response times, which are caused by musicians' greater training of consonant-dissonant judgments (for a discussion, see Bigand et al., 2001), the critical and striking point of the present study is to reveal similar perceptual behaviors in musically trained and untrained listeners within an experimental situation that involved very small musical manipulations (i.e., tonic vs. subdominant functions). Moreover, musically untrained listeners demonstrated robust cognitive priming effects similar to those of musically trained listeners, even when chord sequences were played at an extremely fast tempo. This finding converges with recent electrophysiological evidence showing that nonmusicians' brains process Western musical structures in a sophisticated way (Koelsch, Gunter, Friederici, \& Schroeger, 2000; Maess, Koelsch, Gunter, \& Friederici, 2001) and sometimes very similarly as do musicians' brains (Regnault et al., 2001; Trainor, Desjardins, \& Rockel, 1999; Trainor, McDonald, \& Alain, 2001). As underlined by Trainor et al. (2001), this sophisticated ability to process musical structures in the absence of explicit musical training suggests that the brain places high values on music sounds and is set up for automatically encoding music-specific information. In our view, this sophisticated ability also suggests that nonmusicians' brains are more "musical" than generally assumed.

\footnotetext{
${ }^{7}$ Some repetition blindness effects have nevertheless been consistently reported (Bavelier, 1994; Bavelier \& Potter, 1992; Bavelier, Prasada, \& Segui, 1994).
} 


\section{References}

Bavelier, D. (1994). Repetition blindness between visually different items: The case of pictures and words. Cognition, 51, 199-236.

Bavelier, D., \& Potter, M. C. (1992). Visual and phonological codes in repetition blindness. Journal of Experimental Psychology: Human Perception and Performance, 18, 134-147.

Bavelier, D., Prasada, S., \& Segui, J. (1994). Repetition blindness between words: Nature of the orthographic and phonological representations involved. Journal of Experimental Psychology: Learning, Memory, and Cognition, 19, 1437-1455.

Besson, M., \& Kutas, M. (1993). The many facets of repetition: A cuedrecall and event-related potential analysis of repeating words in same versus different sentence contexts. Journal of Experimental Psychology: Learning, Memory, and Cognition, 19, 1115-1133.

Bharucha, J. J. (1987). Music cognition and perceptual facilitation: A connectionist framework. Music Perception, 5, 1-30.

Bharucha, J. J. (1994). Tonality and expectation. In R. Aiello \& J. Sloboda (Eds.), Musical perceptions (pp. 213-239). Oxford, England: Oxford University Press.

Bharucha, J. J., \& Krumhansl, C. L. (1983). The representation of harmonic structure in music: Hierarchies of stability as a function of context. Cognition, 13, 63-102.

Bharucha, J. J., \& Stoeckig, K. (1986). Reaction time and musical expectancy. Journal of Experimental Psychology: Human Perception and Performance, 12, 403-410.

Bharucha, J. J., \& Stoeckig, K. (1987). Priming of chords: Spreading activation or overlapping frequency spectra? Perception \& Psychophysics, 41, 519-524.

Bigand, E., Madurell, F., Tillmann, B., \& Pineau, M. (1999). Effect of global structure and temporal organization on chord processing. Journal of Experimental Psychology: Human Perception and Performance, 25, 184-197.

Bigand, E., Parncutt, R., \& Lerdahl, F. (1996). Perception of musical tension in short chord sequences: The influence of harmonic function, sensory dissonance, horizontal motion, and musical training. Perception \& Psychophysics, 58, 125-141.

Bigand, E., \& Pineau, M. (1997). Global context effects on musical expectancy. Perception \& Psychophysics, 59, 1098-1107.

Bigand, E., Tillmann, B., Poulin, B., D’Adamo, D., \& Madurell, F. (2001). The effect of harmonic context on phoneme monitoring in vocal music. Cognition, 81, 11-20.

Bigand, E., Tillmann, B., Poulin, B., \& Manderlier, D. (2002). Harmonic priming versus chord repetition effect in music. Manuscript submitted for publication.

Boltz, M. G. (1993). The generation of temporal and melodic expectancies during musical listening. Perception \& Psychophysics, 53, 585-600.

Boltz, M. G., \& Jones, M. R. (1986). Does rule recursion make melodies easier to reproduce? If not, what does? Cognitive Psychology, 18, 389-431.

Bregman, A. S. (1990). Auditory scene analysis: The perceptual organization of sound. Cambridge, MA: MIT Press.

Butler, D. (1989). Describing the perception of tonality in music: A critique of the tonal hierarchy theory and a proposal of intervalic rivalry. Music Perception, 6, 219-242.

Butler, D. (1990). Tonal hierarchies and rare intervals in music cognitionresponse. Music Perception, 7, 325-338.

Chiu, C.-Y., \& Schacter, D. L. (1995). Auditory priming for nonverbal information: Implicit and explicit memory for environmental sounds. Consciousness and Cognition, 4, 440-458.

Cohen, J., MacWhinney, B., Flatt, M., \& Provost, J. (1993). Psyscope: An interactive graphic system for designing and controlling experiments in the psychology laboratory using Macintosh computers. Behavior Research Methods, Instruments and Computers, 25, 257-271.

Cuddy, L. L., \& Lunney, C. A. (1995). Expectancies generated by melodic intervals: Perceptual judgements of melodic continuity. Perception \& Psychophysics, 57, 451-462.

Elman, J. L., \& McClelland, J. L. (1984). The interactive activation model of speech perception. In N. Lass (Ed.), Language and speech (pp. 337-374). New York: Academic Press.

Feustel, T. C., Shiffrin, R. M., \& Salasoo, A. (1983). Episodic and lexical contributions to the repetition effect in word identification. Journal of Experimental Psychology: General, 112, 309-346.

Forster, K. I., \& Davis, C. (1984). Repetition priming and frequency attenuation in lexical access. Journal of Experimental Psychology: Learning, Memory, and Cognition, 10, 680-698.

Fraisse, P. (1967). Psychologie du temps [The psychology of time]. Paris: Presses Universitaires de France.

Francès, R. (1988). The perception of music (J. W. Dowling, Trans.). Hillsdale, NJ: Erlbaum. (Original work published 1958)

Humphreys, G. W., Besner, D., \& Quinlan, P. T. (1988). Event perception and the word repetition effect. Journal of Experimental Psychology: General, 117, 51-67.

Huron, D., \& Parncutt, R. (1993). An improved model of tonality perception incorporating pitch salience and echoic memory. Psychomusicology, $12,154-171$

Jacoby, L. L. (1983). Perceptual enhancement: Persistent effects of an experience. Journal of Experimental Psychology: Learning, Memory, and Cognition, 1, 21-38.

Jacoby, L. L., \& Dallas, M. (1981). On the relationship between autobiographical memory and perceptual learning. Journal of Experimental Psychology: General, 3, 306-340.

Jacoby, L. L., \& Witherspoon, D. (1982). Remembering without awareness. Canadian Journal of Psychology, 36, 300-324.

Jones, M. R., Boltz, M. G., \& Klein, J. M. (1993). Expected endings and judged duration. Memory \& Cognition, 21, 646-665.

Koelsch, S., Gunter, T., Friederici, A. D., \& Schroeger, E. (2000). Brain indices of music processing: "Nonmusicians" are musical. Journal of Cognitive Neuroscience, 12, 520-541.

Krumhansl, C. L. (1990a). Cognitive foundations of musical pitch. New York: Oxford University Press

Krumhansl, C. L. (1990b). Tonal hierarchies and rare intervals in music cognition. Music Perception, 7, 309-324.

Krumhansl, C. L. (1995). Music psychology and music theory: Problems and prospects. Music Theory Spectrum, 17, 53-81.

Krumhansl, C. L., \& Kessler, E. (1982). Tracing the dynamic changes in perceived tonal organization in a spatial representation of musical keys Psychological Review, 89, 334-368.

Leman, M. (1995). Music and schema theory. Berlin, Germany: SpringerVerlag.

Leman, M. (2000). An auditory model of the role of short-term memory in probe tone rating. Music Perception, 17, 437-460.

Lerdahl, F. (1988). Tonal pitch space. Music Perception, 5, 315-345.

Maess, B., Koelsch, S., Gunter, T., \& Friederici, A. D. (2001). "Musical syntax" is processed in the Broca's area: An MEG study. Nature Neuroscience, 4, 540-545.

McClelland, J. L., \& Elman, J. L. (1986). The TRACE model of speech perception. Cognitive Psychology, 18, 1-86.

McClelland, J. L., \& Rumelhart, D. E. (1981). An interactive activation model of context effects in letter perception: Part 1 . An account of basic findings. Psychological Review, 86, 287-330.

Ohnishi, T., Matsuda, H., Asada, T., Aruga, M., Hirakata, M., Nishikawa, M., et al. (2001). Functional anatomy of musical perception in musicians. Cerebral Cortex, 11, 754-760.

Pantev, C., Hoke, M., Lütkenhöner, B., \& Lehnertz, K. (1989, October 27). Tonotopic organization of the auditory cortex: Pitch versus frequency representation. Science, 246, 486-488.

Pantev, C., \& Lütkenhöner, B. (2000). Magnetoencephalographic studies 
of functional organization and plasticity of the human auditory cortex. Journal of Clinical Neurophysiology, 17, 130-142.

Parncutt, R. (1989). Harmony: A psychoacoustical approach. Berlin, Germany: Springer-Verlag.

Parncutt, R., \& Bregman, A. (2000). Tone profiles following short chord progressions: Top-down or bottom-up? Music Perception, 18, 25-58.

Poulin, B., Bigand, E., D’Adamo, D., Madurell, F., \& Tillmann, B. (2000, May). Does musical expertise influence the processing of harmonic structures? Poster presented at The Biological Foundations of Music, New York Academy of Sciences Conference, New York, NY.

Radeau, M., Besson, M., Fonteneau, E., \& Castro, S. L. (1998). Semantic, repetition and rime priming between spoken words: Behavioral and electrophysiological evidence. Biological Psychology, 48, 183-204.

Regnault, P., Bigand, E., \& Besson, M. (2001). Different brain mechanisms mediate sensitivity to sensory consonance and harmonic context: Evidence from auditory event-related brain potentials. Journal of Cognitive Neuroscience, 13, 241-255.

Rumelhart, D. E., \& McClelland, J. L. (1982). An interactive activation model of context effects in letter perception: Part 2. Psychological Review, 89, 60-94.

Schellenberg, E. G. (1996). Expectancy in melody: Test of the implicationrealization model. Cognition, 58, 75-125.

Schmuckler, M. A. (1989). Expectation in music: Investigation of melodic and harmonic processes. Music Perception, 7, 109-150.

Srinivas, K. (1993). Perceptual specificity of nonverbal priming. Journal of Experimental Psychology: Learning, Memory, and Cognition, 19, 582602

Stuart, G. P., \& Jones, D. M. (1995). Priming the identification of environmental sounds. Quarterly Journal of Experimental Psychology: Human Experimental Psychology, 48A, 741-761.

Tekman, H. G., \& Bharucha, J. J. (1992). Time course of chord priming. Perception \& Psychophysics, 51, 33-39.

Tekman, H. G., \& Bharucha, J. J. (1998). Implicit knowledge versus psychoacoustic similarity in priming of chords. Journal of Experimental Psychology: Human Perception and Performance, 24, 252-260.

Thomas, I. B., \& Fitzgibbons, P. J. (1971). Temporal order and perceptual classes. Journal of the Acoustical Society of America, 48, 1010-1013.

Tillmann, B., \& Bharucha, J. J. (2002). Harmonic context effect on temporal asynchrony detection. Perception \& Psychophysics, 64, 640649.

Tillmann, B., Bharucha, J., \& Bigand, E. (2000). Implicit learning of tonality: A self-organizing approach. Psychological Review, 107, 885913.

Tillmann, B., \& Bigand, E. (2001). Global context effect in normal and scrambled musical sequences. Journal of Experimental Psychology: Human Perception and Performance, 27, 1185-1196.

Tillmann, B., \& Bigand, E. (in press). A comparative review of priming effects in language and music. In P. McKevitt, C. Nualláin, \& C. O. Mulvihill (Eds.), Language, vision, and music. Amsterdam: John Benjamins.

Tillmann, B., Bigand, E., \& Pineau, M. (1998). Effects of local and global context on harmonic expectancy. Music Perception, 16, 99-118.

Trainor, L. J., Desjardins, R. N., \& Rockel, C. (1999). A comparison of contour and interval processing in musicians and nonmusicians using event-related potentials. Australian Journal of Psychology, 51, 147-153.

Trainor, L. J., McDonald, K. L., \& Alain, C. (2001). Electrical brain activity associated with automatic and controlled processing of melodic contour and interval. In R. Zatorre \& I. Peretz (Eds.), The biological foundations of music: Annals of the New York Academy of Sciences ( Vol. 930, pp. 429-432). New York: New York Academy of Sciences.

Tulving, E., Schacter, D. L., \& Stark, H. A. (1982). Priming effects in word-fragment completion are independent of recognition memory. Journal of Experimental Psychology: Learning, Memory, and Cognition, 8, 336-342.

Warren, R. (1993). Perception of acoustic sequences. In S. McAdams \& E. Bigand (Eds.), Thinking in sounds: The cognitive psychology of human audition (pp. 37-68). Oxford, England: Oxford University Press.

\section{Appendix}

Number of Identical Notes Shared by the Targets With All 7 Chords of the Previous Contexts and With the 3 Most Recent Chords, Averaged Over All Experimental Sequences

\begin{tabular}{lccccc}
\hline & \multicolumn{2}{c}{ No target in context } & & \multicolumn{2}{c}{ Subdominant in context } \\
\cline { 2 - 3 } \multicolumn{1}{c}{ Chord } & Tonic target & Subdominant target & & Tonic target & Subdominant target \\
\hline All 7 chords & 4.33 & 5.50 & & 5.41 & 6.83 \\
Chord 5 & 0.67 & 1.17 & 1.00 & 1.33 \\
Chord 6 & 0.00 & 1.25 & 0.67 & 1.67 \\
Chord 7 & 0.75 & 0.75 & 0.58 & 0.83 \\
\hline
\end{tabular}

Note. A C major chord consisting of the notes (C2-G2-E3-C4), for example, shares one note with a G major chord consisting of the notes (G1-G2-D3-B3).

Received March 28, 2001 